\title{
GLI SCRITTI ‘ITALIANI’ DI MANUEL LASSALA
}

\author{
MAURIZIO FABBRI (*)
}

SuNTO. - Il saggio indaga sulla influenza esercitata dalla cultura e dalla lingua italiana sull'opera in verso ed in prosa, dell'autore, a lungo vissuto esule in Italia.

$* * *$

ABSTRACT. - This essay inquires the influence exercised by Italian language and culture on the works in verse and in prose written by the author, long lived in our country as an exile.

Tra i numerosi gesuiti spagnoli che si mostrarono letterati in Italia dopo l'espulsione della Compagnia di Gesù imposta da Carlo III di Borbone nel 1767, Manuel Lassala Sangermán si segnala per la varietà degli scritti, ben accolti dal pubblico e dalla critica, e per l'assiduità con cui ha impiegato lingua e vernacoli italiani nella composizione delle sue opere in verso e in prosa.

Non ancora professo, era già noto ai confratelli della natia Valenza per vari componimenti in castigliano, latino e greco, e per due tragedie, di tema biblico e storico, rappresentate e pubblicate.

A Ferrara trovò una prima sistemazione, passando poi a Bologna ove fece professione solenne dei voti e dove trascorse quasi tutti i trentadue anni dell'esilio.

Le doti di verseggiatore e di drammaturgo gli procurarono "buen nombre" presso la società colta felsinea, come non mancò di segnalare nelle Cartas a su hermano ${ }^{1}$ il confratello Juan Andrés, poligrafo dottissimo, autore tra l'altro di una storia universale della letteratura che il

${ }^{(*)}$ Università di Bologna, Italia. E-mail: mrzfabbri@gmail.com

1 J. Andrés, Cartas familiares a su bermano D. Carlos, Madrid, A. de Sancha, 1786, I, p. 8. 
celebre stampatore Bodoni pubblicò a Parma in italiano in sette tomi di grande formato, summa straordinaria di erudizione e vasta sintesi enciclopedica.

Lassala fu gradito ospite di salotti e cenacoli e membro di numerose Accademie, tra cui l'Arcadia romana e degli Intricati bolognese, pregiandosi dell'amicizia di letterati quali Albergati Capacelli, Frugoni, Varano, Bettinelli. Si dedicò allo studio della poesia e delle lingue e frequentò i corsi universitari di Matematica, Astronomia e Fisica sperimentale, quest'ultimo tenuto dalla celebre Laura Bassi.

A Bologna compose odi, egloghe, carmi, epigrammi, sonetti, epitalami, talvolta d'occasione, in latino, italiano, catalano e greco (citerò soltanto il vasto poema in esametri latini Rhenus, apprezzato da Bettinelli e dai recensori del "Nuovo Giornale de' Letterati d'Italia") e tradusse dal francese e dal greco, come il monologo drammatico Pygmalion di Rousseau e le favole attribuite a Lokman, il leggendario poeta dell'Arabia preislamica.

Sempre a Bologna scrisse in italiano, rappresentò con successo e fece pubblicare tragedie ispirate alla classicità greca, come la Ifigenia in Aulide, o suggerite da vicende della storia medievale e dell'epopea della conquista e colonizzazione americana, ed è il caso di Ormisinda, Sancio Garcia, Giovanni Blancas e Lucia Miranda. Compose una sola commedia, Il filosofo moderno. Infine, diede un pregevole contributo alla letteratura odeporica con il poema in endecasillabi sciolti Viaggio da Bologna a Ferrara.

Su queste due ultime opere soffermeremo la nostra attenzione.

Il Viaggio, ${ }^{2}$ dunque. Nella forma metrico-poetica congeniale ai temi didascalico-divulgativi, egli immagina di rivolgersi in forma epistolare al fratello Joaquín, rimasto nella casa paterna a Valenza, per sollecitarlo a fargli visita in Italia, giacché il rimpatrio gli era impedito dalle ordinanze borboniche. Nel comporre il poema si avvale dei ricordi legati alle ripetute visite al contado ferrarese ed alla città, ove poteva contare sull'affetto dei numerosi confratelli che vi si erano stabiliti basterà citare Pedro Montengón, rinnovatore del romanzo spagnolo e sull'amicizia e la stima dei letterati locali. Quei luoghi gli erano cari per più di un motivo: per l'aspetto agreste e pastorale ben accetto al suo

2 M. Lassala, Viaggio da Bologna a Ferrara, a cura di M. Fabbri, Abano Terme, Piovan Editore, 1995. 
gusto ancora parzialmente arcadico e barocco, avvertibile nell'enfasi e sonorità di molti versi ove non mancano diminutivi e vezzeggiativi come "ruscelletto", "Zeffiretto", "capannetta". In secondo luogo, per le singolari analogie con la terra natale: la vasta piana che si affaccia sul mare, lambita qui dall'Adriatico e là dal Mediterraneo; i fiumi che bagnano le città: il Turia a Valenza, il Reno a Bologna, il Po a Ferrara; le lagune e le maremme così somiglianti all'albufera valenzana; le greggi e gli armenti, le capanne dei contadini e dei pastori, immagini comuni alle terre catalane ed emiliane di allora.

Il componimento illustra i diversi momenti dell'escursione a Ferrara e dà conto con erudita puntualità di personaggi, vicende, luoghi e monumenti. Denso di riflessioni personali e di immagini eleganti ed efficaci, che ben si addicono alla facile narratività dell'opera ed all'intento didascalico, il Viaggio offre una molteplicità di situazioni e di toni, che variano dal pastorale all'epico, al patetico e sentimentale, con ampio ricorso a metafore ed allegorie. Non mancano i riferimenti alla realtà politica e sociale del tempo, sostenuti da un diffuso autobiografismo. Lassala, mentre il calesse lo conduce a destinazione, intesse un cauto discorso filosofico e politico, di stampo sostanzialmente conservatore, ma non estraneo alle istanze illuministiche. Così, la contrapposizione virgiliana tra il mondo rurale, inteso come virtuoso e pacifico, e quello cittadino, artificioso e corrotto, si colora di blando rousseaunismo: la natura è amica generosa ("Oh, amabile Natura! Oh come dolci, / Come vivaci sono i puri sensi / De' tuoi piaceri!, vv. 89-91) che elargisce a quanti seguono le sue leggi e vivono dei suoi frutti "l'alma Felicità" (v. 169) che è negata all'"alte regge" (v. 172) ed al "ricco cittadin" (v. 142) al quale "di sfrenato lusso / Gl'inesausti desir, le ambiziose / Mordaci cure" non arrecano che noia e turbamento (vv. 163-165). Ferma è la condanna della guerra, considerata frutto nefasto della rinuncia alla "sovrana Ragion" (v. 601), così come esplicita è l'esaltazione dei vantaggi che comporta la pace, dispensatrice di progresso e benessere.

La sensibilità duttile ed acuta dell'autore sa pervenire ad una gradevole armonia di stili e di linguaggi, ove la lezione rinascimentale e barocca si fonde con quella arcadica e classicheggiante, con il preannuncio di forme che il Romanticismo farà proprie. Così, per esempio, una sintassi poetica da Ariosto 'illuminista' ("Il commercio, il parlar, le armi, i costumi...", v. 40) s'accosta a stilemi tassiani 'arcadici' ("Al biancheggiar nell'oriente il giorno / Già dell'umida notte in ciel cadeano / 
Precipitose l'ombre...", vv. 48-50); versi densi di sensibilità "protoromantico'-illuministica ("I dubbiosi color, che a poco a poco / La tenue luce alle create cose / Rendea, già tolti dall'oscura notte: / Indi crescendo e riflettendo alquanto / Il debol raggio dell'incerto lume, / Vedeasi errante serpeggiar, qual puro / Liquido argento", vv. 59-65) paiono prevalere sui residui arcadici: "Scherzando intorno il Zeffiretto amico / Per entro ad ondeggianti incolte frondi...", vv. 80-81). Echi pariniani ("Sovente il ricco cittadin, fuggendo / E la città e se stesso: il suo soggiorno, / Malgrado il fasto d'un insano lusso...", vv. 142-144) anticipano atmosfere leopardiane ("Talor di rimembrar le ree sventure / Che furo a noi compagne e i lieti giorni / Di richiamar nella smarrita mente /.../ Di nuova sorte lusinghiera speme", vv. 314-319).

La legittimazione del potere temporale dei papi e la difesa apologetica dell'operato dei Legati pontifici non gli fanno dimenticare l'altissimo livello di civiltà raggiunto da Ferrara negli "aurei / Tempi andati" (vv. 327-328) di Alfonso I d'Este né i guasti provocati dalla devoluzione del ducato alla Chiesa in conseguenza del quale "Ben mille / E mille cittadin le patrie mura / Abbandonar, fuggì l'industria seco, / Cadde il lusso, languiro l'arti, muta / La lira giacque polverosa" (vv. 359-363). Non manca il dolente e pur polemico accenno alle drammatiche vicende, definite "importune e aspre" (v. 301), che portarono all'espulsione della Compagnia ed al suo immeritato e prolungato esilio.

La tolleranza e la bonomia che caratterizzano l'opera vengono meno quando si tratta di affrontare questioni di fede o riferibili all'ambito religioso. In tali casi, la più radicale intransigenza porta il poeta ad esaltare la "vera intatta Fede" e l'“aurea Verità" (vv. 492-493) contro ogni altra manifestazione di religiosità. La visita al ghetto di Ferrara, per esempio, lo fa prorompere in invettive di inusitata, intollerante violenza. Gli ebrei ferraresi, che le leggi di allora obbligavano a farsi riconoscere con un drappo giallo che rivestiva parzialmente il capo, divengono "obbrobrio e vituperio delle genti" (v. 421), oppure "mendace turba" (v. 432), "gente vile" (v. 438) che ovunque andasse diffondeva "lo spavento e il terror" (v. 431) tendendo "Con nera frode cieche insidie" (v. 435).

La prima invettiva rimanda all'Alighieri (Inferno, XXXIII, v. 79) al quale Lassala deve più di un verso, come segnalerò nei casi più palesi. Ma non è difficile cogliere echi di sommi lirici spagnoli - Luis de León e Góngora, per esempio - ed italiani, che l'autore elogia citandoli con schietta ammirazione, quali Ariosto, Tasso, Pulci, Guarini, Marino, 
Parini, talora originari di Ferrara, o che ad essa erano collegabili, città di cui conosceva bene la storia ed i meriti culturali, come confermano gli esatti riferimenti ed i pertinenti commenti alle vicende cittadine di maggior rilievo, dall'epoca degli "eterni fasti" (v. 345) di Ercole I e di Alfonso d'Este alla penosa decadenza che aveva fatto seguito all'annessione allo Stato pontificio.

L'ottima conoscenza dei classici latini è attestata dai ripetuti richiami, già a partire dall'epigrafe, a Virgilio, Catullo, Ovidio, con ampie citazioni di miti e divinità dell'Olimpo.

L'influenza dell'antica retorica si manifesta nell'organizzazione stessa dell'opera che presenta una fase introduttiva proemiale, con l'invocazione alla Musa (vv. 1-37); la proposizione, in cui sono esposti gli scopi che l'autore si prefigge (vv. 38-47); l'argomentazione, con la descrizione del viaggio (vv. 48-834); la perorazione finale (vv. 835-842).

Si diceva di Dante. Il v. 10 del Viaggio, "Scorrer le contrade / Del Bel Paese, ch'Appennino parte", riprende la bella e nota espressione dantesca dell'Inferno, xxxiii, v. 80. Il verso 118, "Ecco si rompe / Presso la lunga via da ferree chiostre" rimanda allo stilema tassiano "Ecco apparir Gierusalemme...", assunto nella funzione descrittivo-topografica tipica del poema lassaliano. Altri versi (140-170) che ricordano la vita grama degli infelici contadini ferraresi in opposizione alle comodità dei cittadini ed al lusso ed agli agi dei ricchi riecheggiano l'ideale arcadico sulla opposizione città-campagna già trattato dal Parini nelle Odi - La vita rustica, Il Mattino, del 1768 e 1763 - e dal Pindemonte nelle Prose e poesie campestri (1788). Di ascendenza virgiliana (“...pallor simul occupat ora" (IV, v. 499) è l'accenno al mortale pallore dei coloni vittime delle mefitiche esalazioni palustri (vv. 210-219): "Lenta febbre / Il misero villano ardea sepolto / Nei tuguri, e sedea pallida in viso / Immagine di morte...”.

L'espediente retorico dell'apostrofe dantesca, mediata da Varano e dal Monti, appare nei versi (249-250) dedicati all'ira del fiume Reno imbrigliato da possenti argini: "Va pure, fremi, orgogliosetto fiume, / Minaccia pur..."

Nella descrizione dell'immagine del fiume, che irritato dalle opere di bonifica che disciplinano il suo corso, si erge minaccioso, come il Guadalete del romance medievale di Don Rodrigo, si avvertono echi della Fábula de Polifemo y Galatea di Luis de Góngora: "Polvo el cabello, húmidas centellas destellando...”, vv. 163-165).

Nel rendere omaggio al "ferrarese Omero", v. 277, l'ammirato 
Ludovico Ariosto, ed al suo sepolcro, “... del freddo / Tuo cener muto...", Lassala ricorre alla rielaborazione di un luogo di Catullo "mutam nequiquam adloquerer cinerem". Stilemi e lessico ("cenere muto", "passaggier nasconde al guardo", "marmorea tomba", "l'orme tue", anticipati da Cesarotti e da Young, si ritroveranno in Foscolo e Leopardi risemantizzati da un nuovo afflato preromantico o neoclassico. Ancora stilemi e lessico ("rimembrar le ree sventure", "i lieti giorni", "la smarrita mente", "lusinghiera speme"), paiono anticipare il Leopardi di Silvia.

I versi (584-592) che rievocano gli alloggiamenti militari alla periferia di Ferrara: "Di ben temprato acciaro e ferree canne / Gravide in sen d'arida negra polve / di zolfo mista e d'infiammabil nitro, / Onde al destarsi piccola scintilla / Nella selce percossa, esce rompendo / Precipitosamente il grave piombo/ Cieco ministro d'improvvisa morte..." rimandano alle forti immagini ariostesche dell'episodio di Bireno e dell'archibugio di Cimosco, come si legge nell'Orlando furioso, IX, 91: "O maledetto, o abominoso ordigno, / che fabricato nel tartareo fondo / fosti per man di Belzebù maligno / che ruinar per te disegnò il mondo".

Parafrasi della celeberrima massima "homo homini lupus" attribuita a Thomas Hobbes, devono intendersi i versi "E l'uom nemico / A l'altr'uomo nascosi lacci ordisce", 599). Ed ancora, la leggenda della morte di Fetonte (vv. 720-730), viene riproposta nei termini narrati da Ovidio nelle Metamorfosi, II, di cui riprende taluni versi, così come avviene (vv. 760-775) per la leggenda di Cicni trasformato in cigno (Metamorfosi, VII).

Con frequenza il poeta spagnolo fa ricorso a termini antiquati, come Insubria, Etruria, Eridano.

\section{IL FILOSOFO MODERNO}

Durante il secolo XVIII il teatro - così come le traduzioni, le relazioni di viaggio, i giornali - rappresentò un canale fondamentale attraverso cui la cultura italiana e quella spagnola dialogarono e scambiarono idee, giudizi, tendenze, usi e costumi, migliorando la conoscenza reciproca. Nel nostro Paese, notevole diffusione ebbero il teatro barocco e quello neoclassico spagnolo. Goldoni, e Carlo Gozzi, in alcune loro opere, si ispirarono ai drammi di Lope de Vega, Calderón, Tirso de 
Molina. A loro volta, Metastasio e Goldoni vennero applauditi nei maggiori teatri spagnoli dal pubblico sia tradizionalista sia classicheggiante.

Un importante apporto alla divulgazione in Italia di autori ed opere spagnole venne anche da alcuni gesuiti espulsi che si impegnarono soprattutto nella composizione di tragedie: ricordo, oltre a Lassala, Colomés, Bernardo García, Montengón, Salazar.

Nella composizione del Filosofo moderno, ${ }^{3}$ l'unica commedia sua di cui si abbia notizia, Lassala ebbe presente il teatro goldoniano, anche se nel Prologo afferma di aver tenuto conto pure della lezione di Aristofane, Terenzio e Molière. Numerosi sono i punti di contatto con lo scrittore veneziano, ma vi sono sostanziali differenze per quanto riguarda il tema e le finalità dell'opera.

Fanno pensare al Goldoni l'ambientazione nella Venezia borghese contemporanea; i nomi dei personaggi principali, Rosaura, Lelio, Florindo, Colombina, che ricorrono, per esempio, ne Il bugiardo; il titolo stesso che riprende parzialmente la 'commedia di carattere' Il filosofo inglese.

L'argomento è dato dall'urgenza di maritare Rosaura, donzella ormai trentenne, che corre il rischio di rimanere zitella. Dei due pretendenti che la corteggiano - il giovane Florindo, all'apparenza squattrinato ed il maturo Lelio, all'apparenza saggio e posato - la preferenza della madre di Rosaura, Beatrice, va a quest'ultimo per ragioni suprattutto culturali ed ideologiche. La donna, infatti, che nutre velleità intellettuali, è affascinata dal suo eloquio forbito infarcito di termini francesi, l' 'idioma filosofico" per eccellenza; dal suo posare a filosofo moderno virtuoso e razionale; dai commenti che insieme fanno alle principali opere di Rousseau, Voltaire, Marmontel, Maupertuis. Convinta dalle sentenze di Lelio e dalle letture mal digerite dei philosophes, non si rende conto che il presunto innamorato è un farfallone misantropo ed ipocrita, che disdegna la donna "animal imperfetto" e che non ha alcuna intenzione di prendere in moglie Rosaura. Soltanto l'intervento disinteressato di un amico di famiglia fa sì che venga sbugiardato Lelio e favorito Florindo, al quale un fortunato accadimento ha assicurato il benessere economico.

Il motivo conduttore parrebbe essere, quindi, quello amoroso, ma

3 M. Lassala, Il filosofo moderno, a cura di Joaquín Espinosa, Valencia, Universitat, 1990. 
in realtà, ciò che sta a cuore a Lassala è la denuncia della perniciosa influenza che le dottrine illuministiche - "il veleno che si sparge adesso per ogni dove" (134) - hanno sui giovani e sulle donne che si atteggiano a letterate, corrompendone l'animo ed allontanandole dagli affetti e dalle occupazioni famigliari.

Molto meglio, dunque, pare concludere il messaggio lassaliano ispirato ad un franco conservatorismo, affidarsi al semplice, onesto e "buon" Florindo e salvare 1'"onestade e la innocenza" ispirate alla morale cristiana, assi portanti della famiglia e della società, così "rendendo il vizio ridicolo e la virtù amabile”.

La commedia, in tre atti, è rispettosa delle unità aristoteliche, per cui l'azione è unica e si svolge dal mattino alla sera in un solo ambiente. L'autore si avvale di alcuni espedienti, come gli 'a parte'; la verità carpita con stratagemmi; il ricorso all'assente, vale a dire al personaggio, in questo caso Florindo, che viene evocato, atteso, lodato o biasimato, ma che non giunge mai a calcare la scena.

Abbandonati i toni colti e solenni che caratterizzano i versi del Viaggio, Lassala impiega un linguaggio semplice e piano, di facile comprensione, composto da scambi di battute brevi e rapide, che risultano affettate ed enfatiche solo in alcuni monologhi di Lelio che vi sfoggia la sua erudizione impreziosendola con locuzioni francesi o se ne esce con esclamazioni come "Un certo non so che" ${ }^{4}$ presa di peso dall'opera L'Olimpiade musicata da Antonio Vivaldi su libretto di Metastasio.

Così come l'intreccio della favola risente dell'influenza di modelli appartenenti al teatro spagnolo ed italiano che rimandano, per esempio, al Goldoni ed ai due Moratín, ove non mancano temi come quelli del triangolo amoroso, del matrimonio combinato, della madre saccente, anche il linguaggio presenta una interessante varietà idiomatica, con ispanismi, regionalismi veneti, arcaismi (tipo "onestade").

Numerosi vocaboli sono spagnoli, come vibora, oppure italianizzati come "biblioso", o comuni alle due lingue pur variando l'ambito semantico, come "pretensione", "inavvertenza", "commentario", "repulsiva".

Di impiego frequente sono alcune forme sintattiche spagnole, in

4 Cfr. l'aria per soprano e pianoforte dell'opera: "Un certo non so che / $\mathrm{Mi}$ giunge e passò il cor. / E pur dolor, non è. / Se questo fosse amor? / Nel suo vorace ardor / Già posi incauta, / Posi il piè!". 
specie l'accusativo personale, ("Farò chiamare a Lelio", "lasciar entrare a tutti", etc.), oppure la ripetizione nella stessa proposizione della forma atona e della tonica del pronome personale dativo ed accusativo ("A lei le piacerebbe").

Talune frasi mantengono la costruzione spagnola, come, per esempio, "resterà ubbidita (quedará obedecida)" oppure "che conduce altra volta alla virtù (conduce otra vez a la virtud)" o "poveretti di noi (pobres de nosotros)".

$\mathrm{Al}$ vernacolo veneto rimandano vari termini o espressioni gergali, come "fuora", "morbin", "nisun", "sior" e "siora", "far pulito", "figurarse", "la siora Rosaura stala ben?", con semplificazione delle consonanti doppie ed elisione della vocale finale.

Nelle due opere qui prese in esame si possono riconoscere due registri di scrittura. Nel Viaggio, così come nei componimenti poetici e nelle tragedie, ove sono evocati personaggi eminenti, vicende storiche, opere monumentali, la conoscenza e l'uso della lingua italiana paiono essere pienamente rispondenti alla cultura letteraria dell'autore formatasi sulle pagine dei nostri autori maggiori tanto antichi quanto moderni, mentre nel Filosofo moderno l'argomento popolare, la semplicità dei personaggi che dovevano esprimersi con parole familiari, semplici e spontanee, paiono allentare l'autocontrollo lasciando emergere talune imprecisioni o trascuratezze, qualche carenza lessicale e morfo-sintattica che tuttavia, è bene dirlo, non arrecano danno alla commedia che rimane di piacevole lettura, ben costruita e di sicura efficacia scenica.

Manuel Lassala, dunque, pur rimanendo spagnolo "hasta la médula", come confermano i soggetti delle sue tragedie, l'affiorare costante di riferimenti agli autori del Siglo de oro, la nostalgia per il Paese natio che informa i versi del Viaggio, durante l'esilio ha aderito pienamente alla cultura, alla lingua ed ai dialetti italiani ed ha avuto parte attiva nei fermenti innovatori che animavano quel mondo letterario, fino al punto di non temere di gareggiare con i maggiori drammaturghi italiani con opere del tutto diverse da quelle che venivano rappresentate nei collegi della Compagnia, e di affrontare i rigori della critica con componimenti poetici di elevata ispirazione. Se la penna rimaneva fermamente castigliana, gli inchiostri ove intingerla erano certamente italiani. 